\title{
Effect of antidepressive therapy on retinal contrast processing in depressive disorder
}

\author{
Emanuel Bubl, Dieter Ebert, Elena Kern, Ludger Tebartz van Elst* and Michael Bach*
}

\section{Background}

Recently, we reported a reduced retinal contrast gain in unmedicated and medicated patients with major depression.

\begin{abstract}
Aims
To analyse whether the contrast gain normalises after successful antidepressive therapy by recording the pattern electroretinogram (PERG) in healthy controls and patients with depression before and after antidepressive therapy.
\end{abstract}

\section{Method}

Fourteen patients diagnosed with major depression were repeatedly scanned and the results compared with that from 40 matched controls.

\section{Results}

The retinal contrast gain was lower at baseline in patients with depression, was normalised with remission and correlated with the severity of depression. Patients who did not achieve remission retained significantly lower contrast gain at follow-up.

\section{Conclusions}

The study provides evidence for a state-dependent modulation of retinal contrast gain in patients with major depression. Reduced contrast gain normalised after therapy. A PERG-based contrast gain could serve as a state marker of depression.

\section{Declaration of interest}

None.
For the past few decades there has been an intensive search for biomarkers of psychiatric states such as schizophreniform or depressive syndromes. Currently, different neurobiological signals are being discussed as potential candidates, such as brain volume, cerebral blood flow, plasma proteins, sleep patterns and blood oxygen level-dependent (BOLD) functional magnetic resonance imaging (fMRI). ${ }^{1-5}$ An objective biomarker of depression could be very helpful in distinguishing classical depressive episodes from depression in the context of other disorders such as schizophrenia or life events. It also might be helpful in objectifying the therapeutic response to medication or psychotherapy.

Electrophysiological, psychophysical and fMRI signals arising from the visual system are further candidates as biomarkers of depression. Visual contrast sensitivity and visual-evoked potential amplitudes are known to be reduced in depression. ${ }^{6,7}$ Moreover, there is evidence that antidepressant medication can modulate visually evoked potentials in healthy individuals. ${ }^{7}$ In people with seasonal affective disorder, an initially diminished flash electroretinogram normalised after light therapy, ${ }^{8}$ and mood states have a surprisingly strong influence on the visual-evoked potential. ${ }^{9} \mathrm{~A}$ recently published fMRI study described elevated visual cortical activity in sustained remitted patients with depression and a decrease in activity in those who relapsed. ${ }^{4}$

Another candidate as a biomarker for depressive states is the retinal contrast gain. Retinal contrast processing is easy to assess with an electrophysiological signal, which arises at the level of the retina in response to phase reversing stimuli (gratings or checkerboards) with varying contrasts (Fig. 1). This signal is the pattern electroretinogram (PERG), which reflects retinal ganglion cell activity and is recorded at the cornea in response to visual pattern stimulation. ${ }^{10,11}$ The PERG can thus serve as a surrogate marker of retinal information processing from photoreceptors to the origin of the optic nerve. It is modulated by changes in the catecholaminergic system, especially the dopamine system with a

*These authors contributed equally to the work. reduction under dopamine depletion conditions. ${ }^{12,13}$ Psychopharmacological treatment with antidepressants leads to alterations in the catecholaminergic system that depicts an important pathophysiological mechanism underlining depression. ${ }^{14}$ When stimulating rapidly (faster than about five reversals per second), a somewhat sinusoidal response is obtained in response to the phase-reversing checkerboard. The relationship between the amplitude of this curve and the contrast of the presented grating is nearly linear, i.e. the stronger the contrast, the larger the amplitude. ${ }^{15}$ Since patients do not have to consciously respond in any way but only have to observe the phase-reversing checkerboard, this measurement is objective - a sort of 'electrocardiogram of the eye'.

In a recent study we reported reduced retinal contrast processing as a strong marker of the depressive status in unmedicated and medicated patients. ${ }^{16}$ Figure 1 illustrates typical PERG recordings of participants with current depression and healthy controls in this previous study. The difference in contrast gain between patients and controls was remarkably strong and patients could be distinguished from controls at an individual raw-data level with high sensitivity and specificity. ${ }^{16}$ Thus, the question arises as to whether this easy-to-assess signal might serve as a clinically relevant marker of depression. In particular, the present study examined whether deficient retinal contrast gain (as measured with the PERG) normalises after successful antidepressive therapies or if it remains abnormal even after remission of depression: is the PERG a state marker or a trait marker?

\section{Method}

The basic participant sample for this study is identical to that of our previously published baseline sample: there was no difference between these patients and the patients from the previous study, ${ }^{16}$ which consisted of 40 patients ( 20 in-patients and 20 out-patients) and 40 controls. The 20 in-patients and the 20 out-patients in our previous study both presented with a significant reduction in baseline retinal contrast gain. The baseline contrast gain did not 
differ significantly between the in- and out-patient groups. In absolute figures the out-patient group presented with a slightly, but non-significantly, decreased contrast gain. The 20 out-patients were diagnosed but not treated in our clinic. They were referred to an out-patient psychiatrist for further treatment and were lost to follow-up.

In order to assess treatment effects on the signal of interest, we tried to reassess all 20 in-patients in the initial in-patient sample $(n=20)$. In total, 14 in-patients with a diagnosis of major depression and 40 matched controls (control group) without any psychiatric disease took part in this study. We were unable to reassess the remaining six patients: two were not reassessed as a result of their own decision and four were lost to follow-up. All patients received medication and were investigated at the time of admission and after therapy. The baseline data of the 40 healthy controls and the patients have been published previously. ${ }^{16}$ Here, we report the data of all available follow-up PERG studies to analyse this signal's longitudinal course.

Patients were identified from records in the Department of Psychiatry and Psychotherapy of the University Hospital of Freiburg following approval by the local ethics committee. The study was performed in accordance with the ethical standards laid down in the 1964 Declaration of Helsinki. Patients were included after giving informed consent and were all in-patients admitted to the hospital for further therapy.

Inclusion criteria were a current diagnosis of a major depressive episode according to DSM-IV ${ }^{17}$ criteria classified as major depressive disorder, single episode (DSM-IV: 296.2; 7 patients) or recurrent episode (DSM-IV: 296.3; 7 patients). Exclusion criteria were any other DSM-IV Axis I psychiatric disorder, chronic depression, any other general neurological or medical condition or any eye disease except for correctable refraction errors.

The psychiatric diagnosis was established by senior consultant psychiatrists based on a structured clinical interview following DSM-IV criteria. ${ }^{18}$ In addition, all the patients and the control group were assessed psychometrically using the Beck Depression Inventory $(\mathrm{BDI})^{19}$ and Hamilton Rating Scale for Depression (HRSD) (German translation). ${ }^{20}$ The severity of depression was categorised according to the cut-off levels in the BDI manual (German translation): mild to moderate if the BDI was between 11 and 18 , and moderate to severe if it was greater than $18 .{ }^{19}$ To detect any confounding effects from somatic antidepressant treatment on the target variable, we assessed the medication taken by the patients according to the unipolar antidepressant composite rating (UACR). ${ }^{21,22}$

This study was done in a naturalistic setting, meaning that our measurements coincided with the normal routine treatment of in-patients. The follow-up was conducted at the time of discharge and treatment outcome was evaluated with BDI and HRSD scores. Following the general recommendation for interpretation of BDI and HRSD scores for non-symptomatic states, we defined remission a priori as a BDI of $\leqslant 10$ and a HRSD of $\leqslant 7$.

We set no restrictions with respect to pharmacological or psychotherapeutic therapy in the context of this study. Therefore, the 14 patients' medication varied greatly. The precise medication taken by the patients at baseline was as follows: one patient was on benzodiazepines; two patients underwent monotherapy with mirtazapine or venlafaxine; four patients took a combination of selective serotonin reuptake inhibitors (SSRIs) or venlafaxine with mirtazapine; one received a combination of a tricyclic antidepressant and an SSRI; and six were taking a complex combination of more than two psychotropic drugs. The patients' medication at the follow-up was: one patient was not taking medication; three patients underwent monotherapy with mirtazapine, an SSRI or lithium; one received a combination of a tricyclic antidepressant and venlafaxine; eight were under a complex combination of more than two psychotropic drugs; and one received a combination of an SSRI and lithium.

During the course of the study, four patients received antipsychotic medication; three of these individuals had achieved remission and one had not. Owing to the small patient numbers, we could not further elucidate any possible relationships between antipsychotic medication and contrast gain.

At baseline two people who achieved remission received risperidone; in one person the medication was discontinued and in the other person it was switched to quetiapine. Furthermore, one patient with non-remitted depression received quetiapine at baseline and at follow-up. Finally, one patient received quetiapine at follow-up.

Following UACR criteria, four patients on medication received a score of zero at baseline due to low dosage and short duration of antidepressant medication. Two patients on medication were under moderate-intensity treatment and eight patients underwent high-intensity somatic therapy. Accordingly, at the time of follow-up, one patient was on no medication, two were on moderate treatment and 11 patients received high-intensity somatic treatment (including the four patients with non-remitted depression).

The control group, identical to that of our previous paper, ${ }^{16}$ comprised 40 age- and gender-matched healthy individuals without a history of neurological or mental disorders, all of whom scored within the normal range of the BDI and HRSD. ${ }^{16}$

All participants had a visual acuity above 20/25 wearing appropriate correction, measured at the distance used for visual stimulation. $^{23}$

\section{Stimulation and recording}

Stimulation, recording and analysis were performed using the EP2000 system. ${ }^{24}$ All details were as described in our previous article. ${ }^{16}$ Briefly, in a dimly lit room, the stimuli were generated with a resolution of $800 \times 600$ pixels at a frame rate of $75 \mathrm{~Hz}$ and displayed on a display covering a field size of $32^{\circ} \times 27.0^{\circ}$ at an observation distance of $57 \mathrm{~cm}$ with a mean stimulus luminance of $45 \mathrm{~cd} / \mathrm{m}^{2}$. Our patients were refracted as necessary to the observation distance. To ensure appropriate fixation and accommodation, they reported digits that appeared in random intervals in place of the fixation target.

To assess the PERG-based contrast gain, a sequence of five checkerboard stimuli with 0.8 check size, contrast-reversing at 12 reversals per second, was presented with contrast levels of $3.2 \%, 7.3 \%, 16.2 \%, 36 \%$ and $80 \%$. Each contrast level was presented for $10 \mathrm{~s}$, and then the next contrast was applied, returning finally to the first contrast level. This interleaved sequence was presented until 80 artefact-free trials per contrast (1.0 s length each, containing 12 responses) were accumulated. The interleaved blocking ensured that any sequential effects (for example, fatigue) distributed equally across all contrast values. The protocol was repeated once, and further analysis was based on the vector average of each pair of recordings.

The PERG signals were recorded simultaneously from both eyes using DTL electrodes ${ }^{25}$ placed at the lower limbus of each eye. These were referenced to gold cup electrodes at the ipsilateral outer canthi; one earlobe was grounded. Participants were instructed to blink infrequently during recording and to maintain a relaxed pose. Sweeps exceeding $\pm 130 \mu \mathrm{V}$ were rejected as artefacts, and the number of artefacts per condition was saved with the PERG data. Participants reported small digits appearing 
randomly in place of the fixation cross to ensure correct fixation and accommodation. The test-retest reproducibility of the PERG can be very good with careful electrode placement; when quantified by the coefficient of variation this was reported to be below $10 \%$. $^{26}$

The potentials were amplified, filtered (first order $0.5-100 \mathrm{~Hz}$ ) and digitised at $1 \mathrm{kHz}$ with 16 -bit resolution. To prevent temporal aliasing, all timing (stimulation, analogue sampling, sweep length) was related to the stimulus monitor frame rate. The recording's total duration was approximately $1 \mathrm{~h}$ per participant.

\section{Data analysis}

Offline, all traces (Fig. 1, far left column) were Fourier-analysed to calculate the magnitude spectrum (Fig. 1, right part of the left column, top: the spectrum contains responses at the reversal frequency $(12 \mathrm{~Hz})$ and its harmonics $(24 \mathrm{~Hz}, 36 \mathrm{~Hz})$, noise at all frequencies and occasional isolated mains interference at $50 \mathrm{~Hz}$ ). From this, we extracted a noise-free response estimate. ${ }^{27,28}$ The $12 \mathrm{~Hz}$ response and its harmonic $(24 \mathrm{~Hz})$ were combined via their quadratic mean (root mean square value). A linear model of these spectral response magnitudes $v$. stimulus contrast yielded the PERG-based contrast gain defined by the slope of the linear model. ${ }^{16}$ This slope will be referred to as the 'PERG-based contrast gain' or 'contrast gain' throughout this paper and represents the average from both eyes in each participant. The entire analysis is fully automatic and does not require manual peak measurement, thus no need for masking procedures arose.

\section{Statistical analysis}

The R statistical system ${ }^{29}$ was used by M.B. to analyse the data. Post hoc analysis was performed to test the effects of type of diagnosis and medication. Spearman rank correlations between contrast gain and the BDI and HRSD scores were calculated.

\section{Results}

\section{Electrophysiological findings}

In Fig. 1, the original PERG data from two participants are illustrated. The left column shows that the PERG amplitude rises in response to increasing contrast of the checkerboard stimulus. The middle column demonstrates less increase in PERG amplitude in response to an increase in the stimulus contrast, which is apparent at the raw-data level of an individual patient. The third column illustrates that this 'PERG pathology' normalises in the same patient after remission of depression.

From the original PERG data the contrast gain in $\mu \mathrm{V} / 100 \%$ is derived (Fig. 2(a)). The group comparison revealed a highly significant difference in the contrast gain between healthy controls (left box in Fig. 2(b)) and patients with current depression at baseline (middle box in Fig. 2(b), $P<0.0001$, remaining significant after correcting for multiple testing). The contrast gain of the 10 patients with remitted depression (right box, Fig. 2(b)) did not differ significantly from the controls at follow-up ( $t$-test, $P=0.35)$ - in fact, its median was even a little higher than the median of the controls. In total, 4 of the 14 medicated patients did not remit during the course of the study as indicated by their BDI and HRSD scores (Table 1). The PERG gain of those patients did not differ significantly from the patient group at baseline ( $t$-test, $P=0.96)$. Additionally, at baseline there was no significant difference between the non-remitted and the remitted groups $(P<0.16)$.

In summary, the visual deficit as seen in the PERG normalised with remission. These effects are apparent on an individual basis when comparing the contrast gain at baseline with follow-up (Fig. 3 and Table 2).

\section{Duration of antidepressant therapy}

The duration of antidepressant treatment was highly similar for the two depression groups $(P=0.74)$. For the non-remitted group, the mean treatment time was 12.3 weeks (s.d. $=5.0)$ in contrast to 13.5 weeks (s.d. $=6.4$ ) in the remitted group. Because this study was done in a naturalistic setting, we did not intervene with the individual discharge schedule and ended up with a slightly longer treatment period for the remitted group. In this context four patients were discharged not fully remitted. However, there was no correlation between duration of therapy and contrast gain (rho $(\rho)=0.081, P=0.78$ ). We compared the contrast gain of the four individuals in the non-remitted group with four individuals in the remitted group matched for treatment duration and found again a highly significant difference in contrast gain $(P<0.0003)$. The treatment time for the matched remitted group was 11.8 weeks (s.d. $=5.5)$. The same was true when we matched for age $(P<0.0003)$. The mean age of the matched remitted group was 49.5 years $($ s.d. $=10.8)$.

A linear mixed-effect model for statistical analysis emphasised that the change in contrast gain depended on the status of the patients as remitted, not on the time of investigation. It detected a highly significant difference in contrast gain for the patients with remitted depression $(P<0.001)$. The effect for investigation at baseline or follow-up was not significant, representing the high reproducibility of the result $(P<0.79)$.

\section{Medication effects}

The precise medication taken by the patients at the time of study varied because of our study's naturalistic design. Since most of the 14 patients taking medication received complex antidepressive medication of more than two drugs (see Method), we were unable to identify effects of specific substance classes such as SSRI or tricyclic agents on contrast processing. Furthermore, we observed no significant correlation between intensity of somatic treatment (UACR) and contrast gain. The four individuals in the non-remitted group did not receive any medication not given in the remitted group. In addition, removing the four patients receiving antipsychotic medication did not change the results.

\section{Contrast processing and depressive subsyndrome}

The type of depression - whether recurrent or a first depressive episode - had no effect on the mean contrast gain.

\section{Severity of depression}

To analyse the effect of severity of depression on contrast gain, we compared the BDI and HRSD with the PERG-based contrast gain. The correlation between both BDI and HRSD depression scores and contrast gain across all the control group and patients with depression in the whole patient group and all visits $(\rho=-0.48$, $P<0.0001$ and $\rho=-0.40, P<0.001$, respectively) was highly significant. When looking only at the patient group and combined visits (visit 1 and 2$)$, the results did not differ $(\rho=-0.58$, $P<0.0001$ and $\rho=-0.55, P<0.003)$. The results for the BDI at the follow-up are displayed in Fig. 4.

A receiver operating characteristic (ROC) analysis was performed to assess the robustness of the results: the BDI limit varied from 6 to 20 (bracketing the nominal threshold score of $\leqslant 10$ ) and HRSD from 1 to 20 (bracketing the nominal $\leqslant 7$ threshold score). 

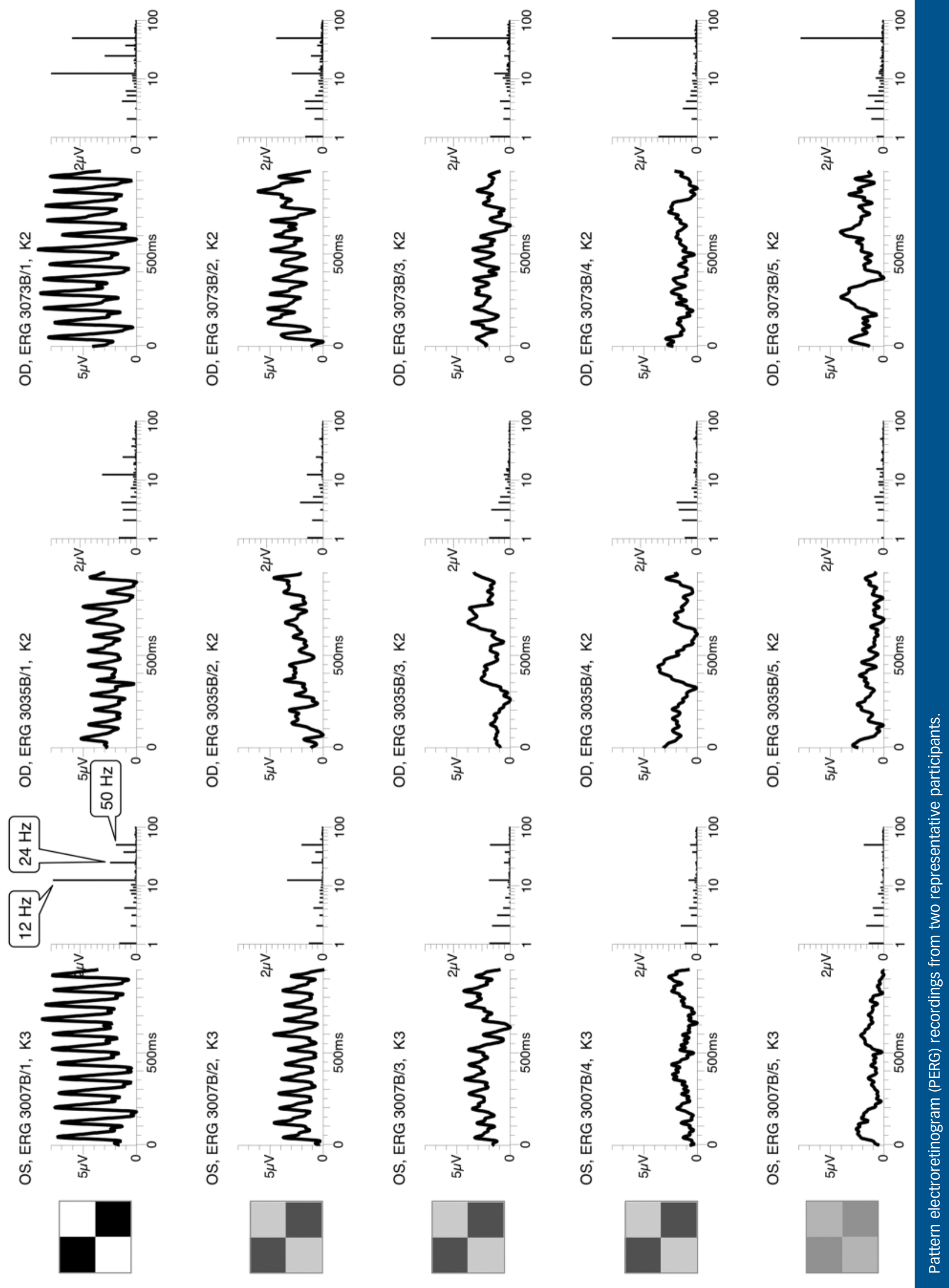

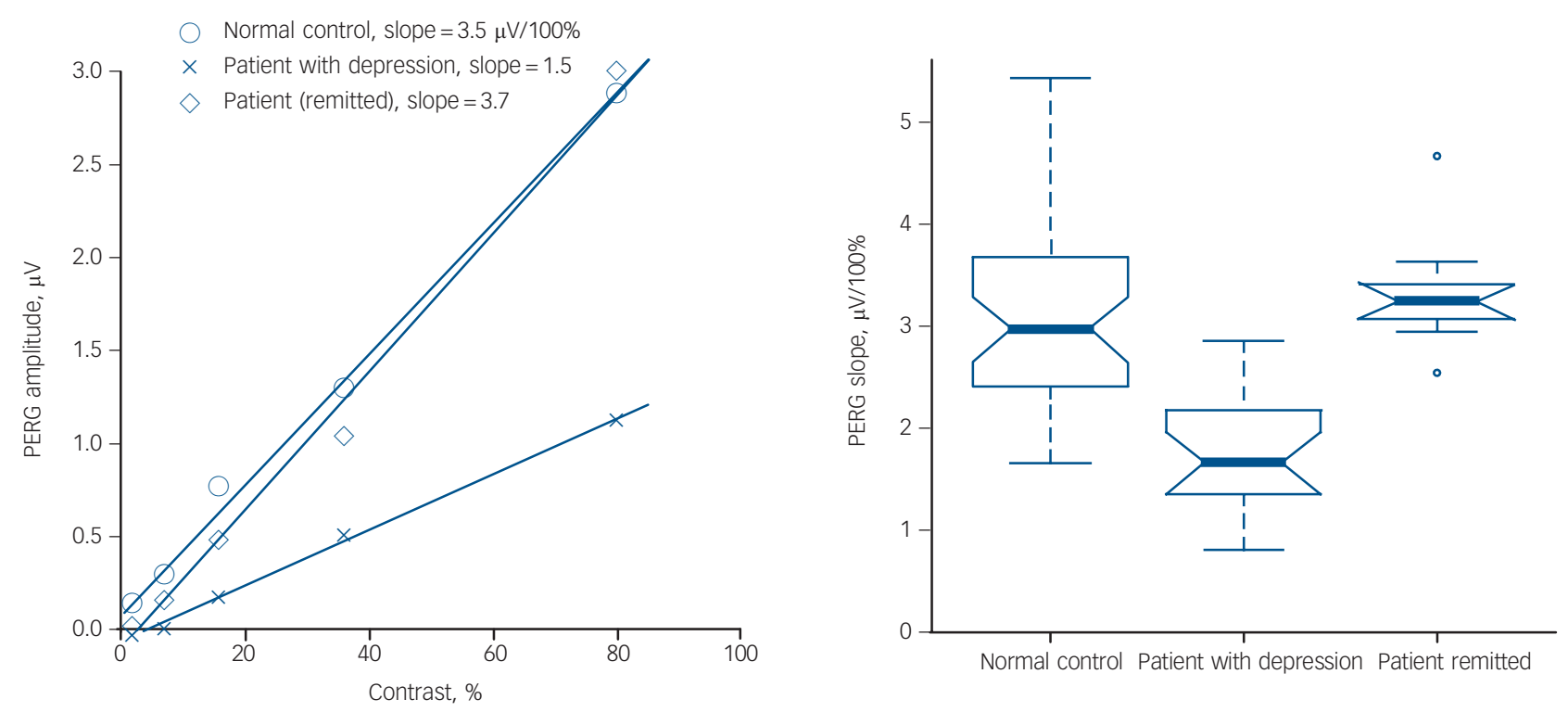

Fig. 2 (a) Pattern electroretinogram (PERG) magnitude $v$. contrast for two participants (a healthy control and a patient with depression before and after remission, single eye) based on the traces in Fig. 1; (b) Group PERG contrast gain, grand average.

In (a) the symbols represent the spectral response magnitude, the oblique lines depict linear fits. The slope of the linear model is interpreted as the PERG-based contrast gain In (b) healthy controls (left) and patients in remission (right) do not differ significantly, whereas patients with current depression present with a significantly reduced contrast gain. Boxplot details on the right: the median is indicated by the thick horizontal lines, the notches represent a $95 \%$ confidence interval for the medians, the box covers the $25-75 \%$ Boxplot details on the right: the median is indicated by the thick horizontal lines, the
percentile range, the 'antennas' indicate the range, outliers are indicated by circles.

\begin{tabular}{|c|c|c|c|c|c|c|c|}
\hline Group & $n$ & $\begin{array}{c}\text { Gender } \\
\text { Male/female, } n\end{array}$ & $\begin{array}{l}\text { Age, years } \\
\text { Mean (s.d.) }\end{array}$ & $\begin{array}{l}\text { Follow-up time } \\
\text { Weeks, mean }\end{array}$ & $\begin{array}{l}\text { BDI score } \\
\text { Mean (s.d.) }\end{array}$ & $\begin{array}{l}\text { HRSD score } \\
\text { Mean (s.d.) }\end{array}$ & $\begin{array}{c}\text { Slope, V/100\% } \\
\text { Mean (s.d.) }\end{array}$ \\
\hline \multicolumn{8}{|l|}{ Patients, visit 1} \\
\hline Later remitted & 10 & $1 / 9$ & $40.2(12.8)$ & & $25.6(7.7)$ & $19.2(6.8)$ & $1.87(0.58)$ \\
\hline Later not remitted & 4 & $2 / 2$ & $48.8(9.8)$ & & 24.5 (7.94) & $17.8(3.4)$ & $1.37(0.59)$ \\
\hline \multicolumn{8}{|l|}{ Patients, visit 2} \\
\hline Remitted & 10 & $1 / 9$ & $40.4(12.8)$ & 10.3 & $7.1(1.8)$ & $4.3(1.6)$ & $3.32(0.55)$ \\
\hline Not remitted & 4 & $2 / 2$ & $48.8(9.8)$ & 7.5 & $18.5(9.4)$ & $9.0(3.9)$ & $1.72(0.28)$ \\
\hline Healthy controls & 40 & $17 / 23$ & $43.3(12.7)$ & & $2.2(1.4)$ & $2.0(1.3)$ & $3.10(0.93)$ \\
\hline
\end{tabular}

The ROC was characterised via its area under the curve (AUC). The results can be summarised as follows: if threshold HRSD is 7 or higher, and BDI between 10 and 11, the AUC is 1.0 , corresponding to $100 \%$ specificity and sensitivity. Lower scores for both parameters lead to a loss of sensitivity and a steady decline of AUC down to the chance level 0.5 for HRSD below 4; which is to be expected. For scores above the optimal region of $\mathrm{HRSD}=7 / \mathrm{BDI}=11$ there is a loss of specificity, but AUC only goes down to 0.77 and the decline is much shallower. These calculations demonstrate that the PERG gain has a finite operating region. Specifically, when the depressive state becomes 'too normal', PERG gain looses sensitivity.

\section{Discussion}

\section{Main findings}

In our previous paper we reported a reduced retinal contrast gain as an objective marker of depression in humans. ${ }^{16}$ The goal of the present study was to investigate the potential differences in this signal in response to successful and unsuccessful therapy. Here we demonstrate a normalisation of what had initially been a reduced retinal contrast gain in patients with depressive disorder after remission from depression.
Although the sample size of our follow-up measurements is small, the results are statistically highly significant. When looking at the individual raw data as illustrated in Figs 3 and 4 it becomes clear that there was a marked improvement in contrast gain in all individuals with remitted depression. The coordinates of contrast change (Fig. 3) clearly separated those whose condition remitted from those whose did not. Figure 4 illustrates that this improvement in contrast gain was accompanied by symptomatic improvement as measured with the BDI.

Thus, the initial reduced contrast gain in the patients normalised in those who remitted, whereas the contrast gain in the non-remitted group remained decreased. The results point to an important and relevant neurophysiological signal with respect to the depressive state.

\section{Neurophysiological interpretation of contrast gain in depression}

The question arises how alterations of contrast gain at the level of the retina could be related to the pathophysiology of depression. Changes in contrast processing have also been found in Parkinson's disease. From animal and human studies there is reliable evidence that contrast processing is altered according to 


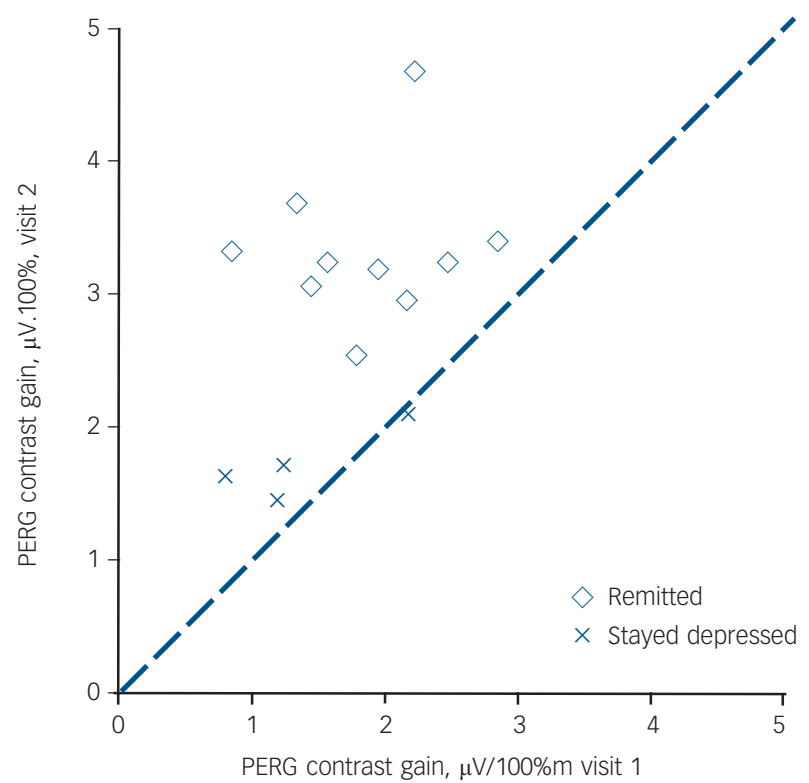

Fig. 3 Relationship between contrast gain at follow-up (visit 2) and baseline (visit 1).

The patients that stayed depressed (indicated by crosses) are near the identity line, whereas those who remitted (diamonds) presented with a significant improvement after remission, and their contrast gain fell within the normal range. PERG, pattern electroretinogram.

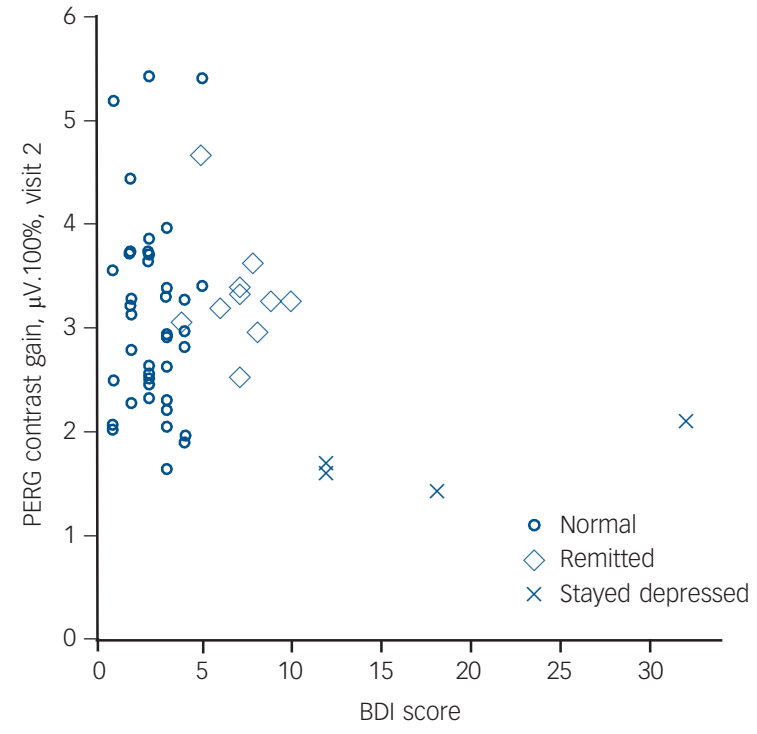

Fig. 4 Correlation between contrast gain and severity of depression (Beck Depression Inventory (BDI) score) at follow-up.

Healthy controls are indicated by small circles, patients in remission by diamonds and patients remaining depressed by crosses. The patients in remission all have a $\mathrm{BDI}$ score $<10$ and their contrast gain fell within the normal range. The four individuals who stayed depressed have a lower contrast gain and, naturally, higher BDI scores. PERG, pattern electroretinogram.

\begin{tabular}{|c|c|c|c|c|c|c|c|}
\hline \multirow[b]{2}{*}{ Patient number } & \multirow[b]{2}{*}{ Gender } & \multicolumn{3}{|c|}{$T_{1}$ (baseline) } & \multicolumn{3}{|c|}{$T_{2}$ (follow-up) } \\
\hline & & $\begin{array}{l}\text { BDI score } \\
\text { Mean }\end{array}$ & $\begin{array}{l}\text { HRSD score } \\
\text { Mean }\end{array}$ & $\begin{array}{l}\text { Contrast gain slope } \\
\mu \mathrm{V} / 100 \% \text {, mean }\end{array}$ & $\begin{array}{l}\text { BDI score } \\
\text { Mean }\end{array}$ & $\begin{array}{l}\text { HRSD score } \\
\text { Mean }\end{array}$ & $\begin{array}{l}\text { Contrast gain slope } \\
\mu \mathrm{V} / 100 \% \text {, mean }\end{array}$ \\
\hline \multicolumn{8}{|c|}{ Non-remitted group } \\
\hline 1 & Female & 28 & 21 & 2.20 & 32 & 14 & 2.10 \\
\hline 2 & Male & 34 & 18 & 1.26 & 12 & 5 & 1.70 \\
\hline 3 & Female & 19 & 13 & 1.21 & 18 & 10 & 1.44 \\
\hline 4 & Male & 17 & 19 & 0.80 & 12 & 7 & 1.61 \\
\hline \multicolumn{8}{|l|}{ Remitted group } \\
\hline 5 & Female & 35 & 33 & 1.59 & 10 & 4 & 3.24 \\
\hline 6 & Female & 12 & 9 & 1.95 & 6 & 1 & 3.19 \\
\hline 7 & Female & 30 & 21 & 2.86 & 7 & 5 & 3.40 \\
\hline 8 & Female & 18 & 23 & 1.44 & 4 & 5 & 3.06 \\
\hline 9 & Female & 29 & 18 & 2.47 & 9 & 7 & 3.25 \\
\hline 10 & Female & 28 & 17 & 1.78 & 7 & 3 & 2.54 \\
\hline 11 & Female & 23 & 13 & 1.35 & 8 & 4 & 3.64 \\
\hline 12 & Female & 20 & 18 & 2.17 & 8 & 5 & 2.95 \\
\hline 13 & Female & 36 & 25 & 2.22 & 5 & 5 & 4.66 \\
\hline 14 & Male & 25 & 15 & 0.89 & 7 & 4 & 3.31 \\
\hline
\end{tabular}

dopaminergic status. ${ }^{12,13,30-33}$ The dopaminergic amacrine cells of the retina are known to be crucially involved in modulating retinal contrast processing. ${ }^{34,35}$ Therefore, we hypothesise that the strong change in the retinal contrast gain is related to dopaminergic dysfunction of the retina.

In Parkinson's disease, dopaminergic disturbance in the visual system is just one aspect of a more widely distributed dopaminergic pathology predominantly affecting the nigro-striatal system. ${ }^{12}$ In analogy, the possible disturbance of dopaminergic neurotransmission in the visual system of people with depressive disorder might represent just one facet of a more widely distributed pathology affecting the systemic dopaminergic system of the brain. Thus, retinal contrast gain might indicate the functional integrity of the systemic dopaminergic system, in particular the mesolimbic branch. This mesolimbic dopaminergic system innervates critical structures of affective information processing such as the amygdala, the nucleus accumbens and the prefrontal brain.

From a number of studies, there is support for an alteration in the dopaminergic system in depressive disorder. ${ }^{36-39}$ Antidepressant treatment has been found to enhance dopaminergic function and influence electrophysiologically measured responses of visual perception..$^{7,40-42}$ The change in visual perception has been reported over a broad variety of antidepressant substance classes or treatment forms. ${ }^{7,8,41}$ 


\section{Limitations}

Due to the naturalistic setting it was not possible to have complete control over all factors and some limitations of the study have to be addressed. Because of the small sample size per medication group, we are not able to comment on any medication effect on the retinal contrast gain due to the lack of respective statistical power. Therefore we cannot comment on whether more adrenergic drugs influenced the improvement in retinal contrast gain more than, for example, serotonergic drugs. Such questions will have to be answered in future studies. Although, both groups did not differ significantly in the mean duration of antidepressant treatment and we compared the 4 individuals with non-remitted depression with 4 participants whose depression remitted with an even shorter duration of antidepressant treatment, the nonremitted group received a shorter period of antidepressant treatment than the remitted group and we cannot fully exclude the possibility that this might have influenced the outcome. Furthermore, we were not able to balance the follow-up time completely.

Both BDI and HRSD scores correlated strongly with the PERG contrast gain. Therefore, we assume that the improvement and normalisation of contrast gain after successful therapy here is a basic correlate of the depressive state. This view is supported by the fact that we did not find any relationship between duration of antidepressant treatment and contrast gain. Thus, the change in contrast gain seems to be physiologically related to the change in severity of depression. This assumption is further supported by findings from an early study where a change in flash-evoked potentials was related to a reduction in severity of depression as measured with the HRSD. ${ }^{43}$

The ROC analysis revealed that the PERG-based contrast gain mirrors depression state best in intermediate depression. In addition, it should be recognised that there are other factors that reduce specificity of PERG-based contrast gain as a state marker: poor optical imaging (wrong glasses) or glaucoma, for instance, also affect the PERG; such confounding factors need to be excluded.

\section{Implications}

In summary, we report a normalisation of an initially reduced contrast gain in patients who remitted from a depressive episode and postulate that contrast gain is a marker of the depressive state. Based on these findings, we hypothesise that retinal contrast gain is an indicator of the functional state of the mesolimbic dopaminergic system, which in turn might be essentially linked to the clinical state of depression. The results are of high relevance if replicated, because the method would provide a marker in animals and humans to investigate treatment success in both established medications and new compounds.

\footnotetext{
Emanuel Bubl, MD, Dieter Ebert, MD, Elena Kern, MD, Ludger Tebartz van Elst $\mathrm{MD}$, Department of Psychiatry and Psychotherapy, Albert-Ludwigs-University of $\mathrm{MD}$, Department of Psychiatry and Psychotherapy, Albert-Ludwigs-University of
Freiburg; Michael Bach, PhD, University Eye Hospital, Albert-Ludwigs-University Freiburg; Michael Bach, PhD,
of Freiburg, Freiburg, Germany

Correspondence: Ludger Tebartz van Elst, Department of Psychiatry and Psychotherapy, Albert-Ludwigs-Universität, Hauptstr. 5, 79104 Freiburg, Germany. Email: tebartzvanelst@uniklinik-freiburg.de

First received 2 Aug 2011, final revision 16 Feb 2012, accepted 3 Apr 2012
}

\section{References}

1 Gong Q, Wu Q, Scarpazza C, Lui S, Jia Z, Marquand A, Huang X, et al. Prognostic prediction of therapeutic response in depression using high-field MR imaging. Neuroimage 2011; 55: 1497-503.
2 Chen Y, Wan HI, O'Reardon JP, Wang DJJ, Wang Z, Korczykowski M, et al. Quantification of cerebral blood flow as biomarker of drug effect: arterial spin labeling phMRI after a single dose of oral citalopram. Clin Pharmacol Ther 2011; 89: 251-8.

3 Domenici E, Willé DR, Tozzi F, Prokopenko I, Miller S, Mckeown A, et al. Plasma protein biomarkers for depression and schizophrenia by multi analyte profiling of case-control collections. PLOS One 2010; 5: e9166.

4 Farb NAS, Anderson AK, Bloch RT, Segal ZV. Mood-linked responses in medial prefrontal cortex predict relapse in patients with recurrent unipolar depression. Biol Psychiatry 2011; 70: 366-72.

5 Landsness EC, Goldstein MR, Peterson MJ, Tononi G, Benca RM. Antidepressant effects of selective slow wave sleep deprivation in majo depression: a high-density EEG investigation. J Psychiatr Res 2011; 45: 1019-26.

6 Bubl E, Tebartz van Elst L, Gondan M, Ebert D, Greenlee MW. Vision in depressive disorder. World J Biol Psychiatry 2009; 10: 377-84.

7 Normann C, Schmitz D, Fürmaier A, Döing C, Bach M. Long-term plasticity of visually evoked potentials in humans is altered in major depression. Biol Psychiatry 2007; 62: 373-80.

8 Lavoie M-P, Lam RW, Bouchard G, Sasseville A, Charron M-C, Gagné A-M, et al. Evidence of a biological effect of light therapy on the retina of patients with seasonal affective disorder. Biol Psychiatry 2009; 66: 253-8.

9 Joost W, Bach M, Schulte-Mönting J. Influence of mood on visually evoked potentials: a prospective longitudinal study. Int J Psychophysiol 1992; 12: 147-53.

10 Maffei L, Fiorentini A. Electroretinographic responses to alternating gratings before and after section of the optic nerve. Science 1981; 211: 953-5.

11 Bach M, Hoffman M. The origin of the pattern electroretinogram (PERG). In Principles and Practice of Clinical Electrophysiology of Vision (eds J Heckenlively, G Arden): 185-96. MIT Press, 2006.

12 Tebartz van Elst L, Greenlee MW, Foley JM, Lucking CH. Contrast detection, discrimination and adaptation in patients with Parkinson's disease and multiple system atrophy. Brain 1997; 120 (Pt 12): 2219-28.

13 Ghilardi MF, Marx MS, Bodis-Wollner I, Camras CB, Glover AA. The effect of intraocular 6-hydroxydopamine on retinal processing of primates. Ann Neurol 1989; 25: 357-64.

14 Hasler G, Fromm S, Carlson PJ, Luckenbaugh DA, Waldeck T, Geraci M, et al. Neural response to catecholamine depletion in unmedicated subjects with major depressive disorder in remission and healthy subjects. Arch Gen Psychiatry 2008; 65: 521-31.

15 Ben-Shlomo G, Bach M, Ofri R. Temporal and spatial frequencies interact in the contrast transfer function of the pattern electroretinogram. Vision Res 2007; 47: 1992-9.

16 Bubl E, Kern E, Ebert D, Bach M, Tebartz van Elst L. Seeing gray when feeling blue? Depression can be measured in the eye of the diseased. Biol Psychiatry 2010; 68: 205-8.

17 American Psychiatric Association. Diagnostic and Statistical Manual of Mental Disorders (4th edn) (DSM-IV). APA, 1994.

18 Sheehan DV, Lecrubier $\mathrm{Y}$, Sheehan $\mathrm{KH}$, Amorim $\mathrm{P}$, Janavs J, Weiller $\mathrm{E}$, et al. The Mini-International Neuropsychiatric Interview (M.I.N.I.): the development and validation of a structured diagnostic psychiatric interview for DSM-IV and ICD-10. J ClinPsychiatry 1998; 59 (suppl 20): 22-33.

19 Hautzinger M, Bailer M, Worrall H, Keller F. Das Beck-Depressions-Inventar (BDI). Überarbeitet und ergänzte Neuauflage. Hans Huber, 1995.

20 Collegium Internationale Psychiatriae Scalarum. Internationale Skalen für Psychiatrie. Beltz Test, 1977.

21 Keller MB. Undertreatment of major depression. Psychopharmacol Bull 1988; 24: $75-80$.

22 Leon AC, Solomon DA, Mueller TI, Endicott J, Rice JP, Maser JD, et al A 20-year longitudinal observational study of somatic antidepressant treatment effectiveness. Am J Psychiatry 2003; 160: 727-33.

23 Bach M. The Freiburg Visual Acuity Test - variability unchanged by post-hoc re-analysis. Graefes Arch Clin Exp Ophthalmol 2007; 245: 965-71.

24 Bach M. Freiburg Evoked Potentials. Bach, 2000 (http://www.michaelbach. de/ep2000/index.html).

25 Bach M. Preparation and Montage of DTL-Electrodes. University of Freiburg, 2007. (http://www.uniklinik-freiburg.de/augenklinik/live/homede/mit/bach/ ops/dtl_en.html).

26 Otto T, Bach M. Retest variability and diurnal effects in the pattern electroretinogram. Doc Ophthalmol 1996; 92: 311-23.

27 Bach M, Meigen T. Do's and don'ts in Fourier analysis of steady-state potentials. Doc Ophthalmol 1999; 99: 69-82.

28 Meigen T, Bach M. On the statistical significance of electrophysiological steady-state responses. Doc Ophthalmol 1999; 98: 207-32. 
29 R Development Core Team. R: A Language and Environment for Statistical Computing. R Foundation for Statistical Computing, 2010.

30 Bodis-Wollner I, Yahr MD. Measurements of visual evoked potentials in Parkinson's disease. Brain 1978; 101: 661-71.

31 Bodis-Wollner I, Marx MS, Mitra S, Bobak P, Mylin L, Yahr M. Visual dysfunction in Parkinson's disease. Loss in spatiotemporal contrast sensitivity. Brain 1987; 110: 1675-98.

32 Glover A, Ghilardi MF, Bodis-Wollner I, Onofrj M. Alterations in event-related potentials (ERPS) of MPTP-treated monkeys. Electroencephalogr Clin Neurophysiol 1988; 71: 461-8.

33 Langheinrich $\mathrm{T}$, Tebartz van Elst, Lagrèze WA, Bach $\mathrm{M}$, Lücking $\mathrm{CH}$, Greenlee MW. Visual contrast response functions in Parkinson's disease: evidence from electroretinograms, visually evoked potentials and psychophysics. Clin Neurophysiol 2000; 111: 66-74.

34 luvone PM, Galli CL, Garrison-Gund CK, Neff NH. Light stimulates tyrosine hydroxylase activity and dopamine synthesis in retinal amacrine neurons. Science 1978; 202: 901-2.

35 Djamgoz MB, Hankins MW, Hirano J, Archer SN. Neurobiology of retinal dopamine in relation to degenerative states of the tissue. Vision Res 1997; 37: 3509-29.

36 Ebert D, Feistel H, Kaschka W, Barocka A, Pirner A. Single photon emission computerized tomography assessment of cerebral dopamine D2 receptor blockade in depression before and after sleep deprivation-preliminary results. Biol Psychiatry 1994; 35: 880-5.

37 Hamilton JP, Chen G, Thomason ME, Schwartz ME, Gotlib IH. Investigating neural primacy in major depressive disorder: multivariate Granger causality analysis of resting-state fMRI time-series data. Mol Psychiatry 2011; 16: $763-72$

38 Saijo $\mathrm{T}$, Takano A, Suhara $\mathrm{T}$, Arakawa $\mathrm{R}$, Okumura $\mathrm{M}$, Ichimiya $\mathrm{T}$, et al. Electroconvulsive therapy decreases dopamine $D_{2}$ receptor binding in the anterior cingulate in patients with depression: a controlled study using positron emission tomography with radioligand $\left[{ }^{11} \mathrm{C}\right] \mathrm{FLB} 457$. J Clin Psychiatry 2010; 71: 793-9.

39 Pei L, Li S, Wang M, Diwan M, Anisman H, Fletcher PJ, et al. Uncoupling the dopamine D1-D2 receptor complex exerts antidepressant-like effects. Nat Med 2010; 16: 1393-5.

40 Schilström B, Konradsson-Geuken A, Ivanov V, Gertow J, Feltmann K, Marcus $\mathrm{MM}$, et al. Effects of S-citalopram, citalopram, and R-citalopram on the firing patterns of dopamine neurons in the ventral tegmental area, N-methyl-Daspartate receptor-mediated transmission in the medial prefrontal cortex and cognitive function in the rat. Synapse 2011; 65: 357-67.

41 Seggie J, Canny C, Mai F, McCrank E, Waring E. Antidepressant medication reverses increased sensitivity to light in depression: preliminary report. Prog Neuropsychopharmacol Biol Psychiatry 1989; 13: 537-41.

42 Volkow ND, Wang G-J, Telang F, Fowler JS, Logan J, Wong C, et al. Sleep deprivation decreases binding of [11C]raclopride to dopamine D2/D3 receptors in the human brain. J Neurosci 2008; 28: 8454-61.

43 Friedman J, Meares R. The effect of placebo and tricyclic antidepressants on cortical evoked potentials in depressed patients. Biol Psychol 1979; 8: 291-302.

\section{Easy Listening}

\section{Dino Campana}

I seek no peace, and cannot stand war I dream my way around the world, quiet and alone

Brimming with muted songs. I long

For the fog and silence of a great port

A great port crowded with light sails

Ready to leave towards the blue horizon

Wavering softly as the whispering wind rises

And sings its brief chords on its way.

And those chords are carried by the wind

Far away over the unknown sea

- I'm dreaming. Life is sad and I'm alone

When, when will my soul awaken in the sun

Shivering with freedom in a morning bright with fire

Awaken in the sun, the eternal sun.

This poem is from Dino Campana's (1885-1932) Selected Works, translated by Cristina Viti and published by Survivors' Poetry in 2006. Dino Campana was admitted to San Salvi, an asylum in Florence at the age of 32 years and transferred to Castel Pulci, a place designated for those regarded as incurable in April 1918 where he remained until his death.

Chosen by Femi Oyebode. 